\section{Macrophage LXRs inhibit atherosclerosis}

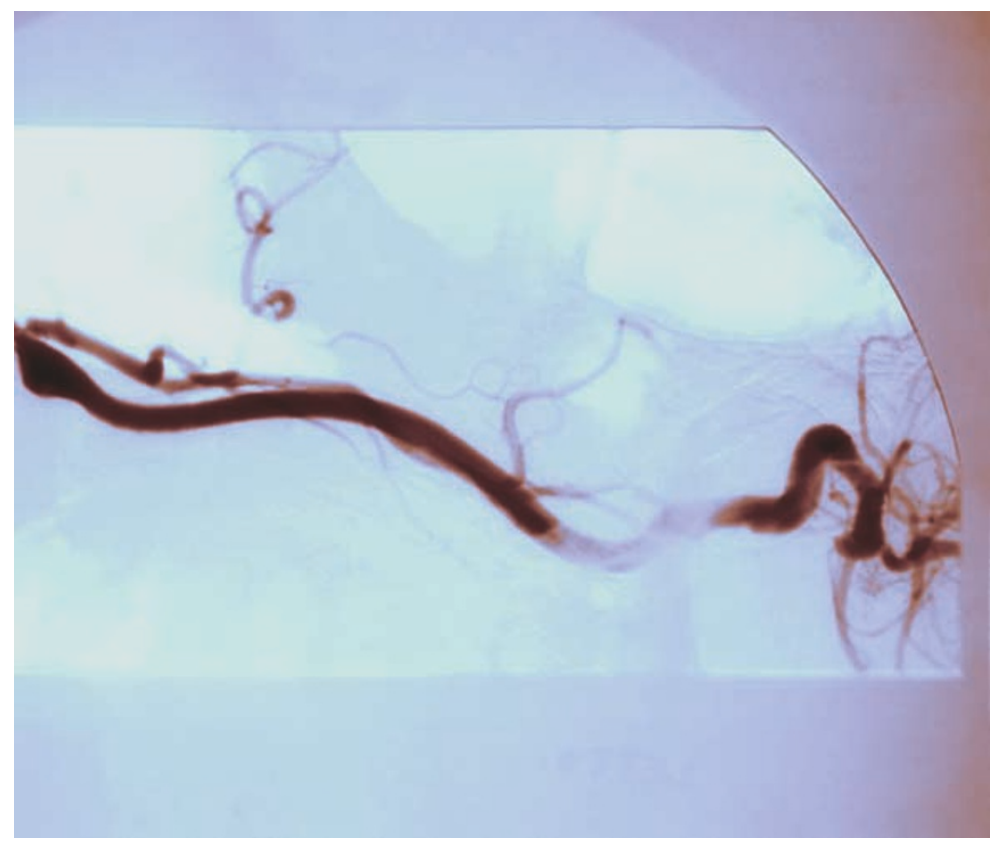

Recent studies have identified the liver X receptor- $\alpha$ (LXR- $\alpha$ ) and LXR- $\beta$ as important transcriptional regulators of cholesterol metabolism and transport. In Proceedings of the National Academy of Science, Tangirala et al. show that LXRs expressed by macrophages protect against atherosclerosis in mouse models of the disease.

LXRs were first identified in the liver, and they are members of the nuclear hormone receptor superfamily of transcription factors. They bind and are activated by cholesterol, whereupon they are involved in regulating the expression of genes involved in sterol absorption and transport, and several genes involved in cholesterol and fatty-acid metabolism. However, LXRs are also expressed in non-hepatic cells, and are involved in regulating the expression of ATPbinding cassette $1(\mathrm{ABC} 1)$ and apolipoprotein E; proteins that have important functions in promoting the transfer of cholesterol to receptors, such as high-density lipoprotein (HDL), in a process known as reverse cholesterol transport. The importance of this process of reverse transport can be seen in the rare genetic condition Tangier disease, in which patients do not express $\mathrm{ABC} 1$. The patients have reduced HDL levels, they accumulate cholesterol in tissues and have an increased risk of atherosclerosis. As LXRs are expressed on macrophages - a cell type that is involved in the formation of atherosclerotic plaques — the authors sought to identify a direct link between LXR signalling from macrophages and the pathogenesis of cardiovascular disease.

To investigate the role of LXR expression on macrophages in atherosclerosis, macrophage-selective knockout mice were created. The use of selective knockouts is a useful technique to investigate a cell-specific contribution without interference of the loss of function in other tissues. Bone marrow from Lxr-deficient mice was transferred to lethally irradiated mouse models of atherosclerosis. The recipient mice showed selective loss of LXR activity in bone-marrowderived cells, increased cholesterol accumulation and accelerated atherosclerosis. These results show a direct

\title{
AUTOIMMUNE DISEASES
}

\section{Lesions with a jagged edge}

What is good for the embryo is not necessarily good for the adult, as a new report in Nature Medicine illustrates. In this paper, John et al. show that a signalling pathway that controls oligodendrocyte maturation in the embryo might contribute to the pathogenesis of multiple sclerosis (MS) if it is reactivated in the adult.

MS is an inflammatory disease that causes progressive demyelination in the central nervous system. This initially causes a deficit in axonal conduction, and unless remyelination occurs, the axons eventually degenerate because they lack the trophic support that myelin provides. In the early stages of the disease, the lesions are repaired quite efficiently, but the capacity for remyelination declines with time. However, even the most advanced lesions contain oligodendrocyte precursors that should be able to repair the damage, so why do they lose this ability in the later stages of MS?
The authors considered what other factors at the lesion site might be interfering with remyelination. The cytokine TGF- $\beta 1$ (transforming growth factor- $\beta 1$ ) is known to be present, and reactive astrocytes have also been implicated in the pathogenesis of MS. To examine how these components might interact to prevent remyelination, John et al. used microarray analysis to find out how TGF- $\beta 1$ affects the gene-expression profile of astrocytes in vitro.

One factor that was found to be upregulated in the presence of TGF- $\beta 1$ was a protein called jagged 1 . During normal development, jagged 1 acts as a ligand for Notch, which is expressed on the surface of immature oligodendrocytes. Binding of jagged 1 to Notch activates the expression of the basic helix-loop-helix transcription factor Hes5 in the oligodendrocyte precursors, and this prevents them from differentiating too early. The authors found that jagged 1 was expressed in active demyelinating lesions, but not in lesions in which remyelination was successful. This indicates that the jagged-Notch-Hes5 pathway is likely to be one of the factors that prevent the oligodendrocyte precursors in MS lesions from acquiring a mature myelinating phenotype.

Although TGF- $\beta 1$ is deleterious in terms of remyelination, blocking its activity altogether is not a viable option, because it also protects against inflammation. These new findings raise the possibility of intervention further downstream - for example, by interfering with Notch signalling — and this could lead to the development of new therapeutic strategies for the treatment of MS.

Heather Wood

Associate Editor, Nature Reviews Neuroscience

(6) References and links

ORIGINAL RESEARCH PAPER John, G. R. et al. Multiple sclerosis: re-expression of a developmental pathway that restricts oligodendrocyte maturation. Nature Med. $\mathbf{8}$, 1115-1121 (2002)

FURTHER READING Franklin, R. J. M. Why does

remyelination fail in multiple sclerosis? Nature Rev. Neurosci. 3, 705-714 (2002)

WEB SITE

National Multiple Sclerosis Society:

http://www.nationalmssociety.org/ 